\title{
Viejas prácticas, nuevos métodos: El diseño digital
}

\author{
Past practices, new methods: The digital design
}

<RESUMEN>

En noviembre de 1849, un año después de haber arribado a Chile el arquitecto francés Claude Francois Brunet de Baines, por encargo del Presidente Montt, da inicio en la Universidad de Chile al primer curso de la carrera de arquitectura en nuestra universidad. Desde esa fecha, las matemáticas superiores y la geometría descriptiva han formado parte de los planes de estudios de la carrera. Este artículo analiza la evolución del foco central de investigación de los programas CAD, y su incidencia tanto en la formación del arquitecto, como en su ejercicio profesional.

El contenido de los planes de estudios se ha visto afectado por los continuos progresos de la ciencia y la tecnología. El actual desarrollo de las tecnologías CAD obliga a la convergencia de varias ciencias, entre ellas biología, epistemología, informática, programación y geometrías computacionales, importantes para la comprensión de los nuevos modos de producción de la arquitectura.

$<A B S T R A C T>$

In November 1849, a year after arriving in Chile, the French architect Claude Francois Brunet de Baines, having been commissioned by President Montt opened at the University of Chile, the first course of the career of architecture. Since then, higher mathematics and descriptive geometry have been part of the curricula of the school. This article analyzes the evolution of the central focus of inquiry of the caD programs, and its impact, both in the formation of the architect, as well as in their professional practice.

The content of the curriculum has been affected by the continued progress of science and technology. The current development of caD technologies requires the convergence of various sciences, including biology, epistemology, computer science, programming and computational geometry, important for the understanding of the new modes of production of architecture.

<PALABRAS CLAVE>

EDUCACIÓN CAD / GEOMETRÍA COMPUTACIONAL / COLABORACIÓN / DISEÑO PARAMÉTRICO / SISTEMAS EVOLUTIVOS

-KEY WORDS

CAD EDUCAT ION / COMPUTER GEOMETRY /

COLLABORATION / PARAMETRIC DESIGN / EVOLUT IONARY SYSTEMS

\section{La razón geométrica}

A finales del siglo xvı, con la constitución de la «nueva ciencia» físico-matemática y en contraste con los planteamientos aristotélicoescolásticos, aparece un nuevo tipo de racionalidad de la que los coetáneos destacan la matematización como su característica más evidente y diferencial. Como por entonces la parte más sólida y valorada de la matemática todavía era el modelo axiomático-sintético de la geometría de Euclides ${ }^{1}$, se la denomina con el término «razón geométrica». Se trataba de un ambicioso programa racional que a partir de las aplicaciones más concretas y mecánicas pretendía elevarse a desarrollos claramente metafísicos, ontológicos o incluso éticopolíticos.

Jean-Nicolas Louis Durand ${ }^{2}$ tuvo una profunda influencia para la arquitectura universal de los pasados siglos XVIII y xIx. El tuvo una cátedra en la École Polytechnique en París, entre los años 1795 a 1830, siendo el primer arquitecto en dictar una cátedra en la Escuela de Ingeniería y Matemáticas ${ }^{3}$. Durante este período realizó

\footnotetext{
Arquitecto y académico Facultad de Arquitectura y Urbanismo de la Universidad de Chile; Departamento de Ciencias de la Construcción.

Matemático griego (330 a.C. - 275 a.C.). Autor de Los elementos, obra en la que se presenta, el estudio de las propiedades de líneas y planos, círculos y esferas, triángulos y conos, etc.; es decir, de las formas regulares.

2 Arquitecto y teórico del arte francés (1760-1834). Autor de la obra Compendio de lecciones de arquitectura ejerciendo una importante influencia en la teoría y en la práctica de la arquitectura, haciendo prevalecer los parámetros de funcionalidad por sobre cualquier otra consideración de tipo estética.

3 Damisch, H. The origin of perspective. Cambridge, MA, EE.UU.: The MIT Press, 1994.
} 
un aporte fundamental para el desarrollo de la arquitectura de los siglos venideros: El traslado de la Geometría Descriptiva del matemático Gaspar de Monge ${ }^{4}$, su colega en la École Polytechnique, a un método de diseño arquitectónico. La transformación de la filosofía de Descartes ${ }^{5}$, en un sistema de coordenadas ortogonales para definir las plantas, cortes y elevaciones, constituyó una idea tan poderosa, que hasta el día de hoy sigue vigente.

Es por consiguiente interesante identifica una arquitectura que es posterior a la teoría de Durand o posterior al movimiento moderno o incluso posterior a las reglas de cualquier geometría. En el número del magazine AD Architectural Design dedicado al tema «After Geometry " ${ }^{6}$, Peter Davidson y Donald Bates en su editorial, exponen una teoría la cual excluye a Descartes y Durand. Adherir a esta posición, no implica abandonar la práctica tradicional de la arquitectura, ni propone, ni supone, el fin de la geometría en la arquitectura. After Geometry es simultáneamente todo lo resultante de vínculo entre arquitectura y geometría, además de la incorporación y expansión de nuevas técnicas y estrategias para una nueva organización y orden de lo arquitectónico. Sin embargo existen algunas inconsistencias con estas «nuevas técnicas y prácticas» de las cuales se pueden señalar algunas, no con el propósito de desarrollar una discusión sino ayudar a comprender si los resultados producidos con el uso de tecnologías computacionales, son realmente nuevos. De ser así ¿por qué la mayoría de la arquitectura generada mediante el uso de computadores todavía sigue representándose en plantas, cortes y elevaciones? ¿Por qué, a pesar del uso de los CAD, todavía es más fácil proyectar un cubo que un paraboloide hiperbólico? ¿Por qué aún la arquitectura generada mediante computadores está fuertemente influenciada por las coordenadas rectangulares y el ángulo recto?

Hasta el Renacimiento, el «arte de las matemáticas» estuvo siempre conectado a la arquitectura; genios matemáticos, como Rafael Alberti o Bruneleschi, son prueba de esto.

Pero, no obstante los grandes descubrimientos hechos por los matemáticos, en comparación con el desarrollo y el aporte de la ciencia durante estos siglos, la expresión de la arquitectura construida, se habría mantenido más menos sin grandes variaciones. Sin embargo, la geometría contiene enormes potencialidades para desarrollar una expresión propia del avance tecnológico materializado en las relaciones espaciales con el entorno y con el medio ambiente en general. Pero hasta finales del siglo xIX, sólo la geometría euclidiana era considerada como la representación correcta del espacio y el ejemplo último de un sistema lógico deductivo ${ }^{7}$.

Muchos teóricos del período neoclásico consideraban la época del Barroco como posiblemente la más extraordinaria en cuanto a genialidad artística, en especial en la arquitectura, entonces aún confundida con la ingeniería y siempre muy próxima a la escultura. Sin embargo, los más críticos y rigurosos, veían en ella la antítesis del racionalismo debido a la incomprensión de su extrema originalidad plástica. Sin embargo, el barroco, encierra un virtuosismo profundo preocupado por la efectividad de la obra sobre los sentidos del individuo, el que no queda confinado exclusivamente en el Arte, sino que presenta fenómenos tan nítidamente vinculados a la razón contemporánea como la tecnología o los medios, cada vez más sensibles a los efectos especiales y a la satisfacción sensorial. Los patrones formales, aunque específicos de una época como el Barroco, supieron derivar la arquitectura hacia una condición de espectáculo dinámicogeométrico que culmina con brillantez la última de las etapas artesanales en la historia del arte constructivo, la de la composición cuidada y la estereotomía de la piedra.

Desde un punto de vista formal, Gerard Desargues $^{8}$, fue reconocido como uno de los más brillantes geómetras de su tiempo, focalizó su interés, contrariamente a Descartes, a definir un método universal para controlar geométricamente el espacio en un orden absoluto. Él fue el primero en comprender (o interpretar geométricamente) que el sol estaba a una distancia infinita, por lo cual, los rayos del sol reprensentaban proyectantes paralelas, y explicaban como dibujar las sombras reales proyectadas por el sol en una pintura. Su sofisticado método de estereotomía, otra de sus áreas de interés, pudo ser aplicado al diseño y construcción de toda clase de

\footnotetext{
Matemático francés (1746-1818). Considerado el invenstor de la geometría descriptiva.

Filósofo, matemático y científico francés (1596-1650). Autor de la obra Discurso del método, y considerado como el padre de la filosofía moderna.

Davidson, P., Bates, D. After geometry. ad-magazines/journals, 1998

Damisch, H. Op. cit.

8 Matemático e ingeniero francés (1591-1661). Creador de la Geometría Proyectiva.
}

bóvedas. La nueva geometría de Desargues lo condujo a establecer que las proyecciones ortogonales eran simplemente un caso particular del método de la perspectiva. Sin embargo, no todos apreciaban las teorías de Desargues, principalmente debido a su concepción acerca del infinito, y sus innovaciones en el campo de la geometría pura.

Es así como la cualidad dimensional del espacio formulada por Descartes, derivó en el más importante descubrimiento del siglo xvII, la Geometría Descriptiva, esto con la concurrencia de tres aspectos principales: El álgebra, que pudo tener una representación física en el espacio; las formas que pudieron ser descriptivas a través del análisis; y la concreción, durante los siglos XVIII y XIX de la mayor parte de las definiciones de la geometría. Precisamente por el dominio de una razón geometrizante profundamente anticualitativa, por ejemplo, el espacio y el tiempo perderán para la razón geométrica sus dimensiones de la vida y cualitativas, para ser identificados reductivamente con las coordenadas matemáticas, homogéneas y meramente cuantitativas, es decir la extensión como cualidad primaria. Estas nuevas tecnologías de representación gráfica que emergieron durante el período enmarcado entre los años 1795 a 1840, pueden ser clasificadas en tres ramas:

a. Investigaciones acerca de sistemas de proyecciones en general, incluyendo las proyecciones ortogonales y sistemas de perspectivas cónicas, con la finalidad de enseñar dibujo geométrico técnico.

b. Investigaciones acerca de la naturaleza de la visión, la cual culmina con la invención de los nuevos sistemas de perspectiva lineal en 1853.

c. Investigaciones en mira a encontrar sistemas gráficos, los cuales estarían optimizados para el uso de las comunicaciones al tiempo que buscarían la simpleza y la facilidad del aprendizaje operacional.

No es circunstancial que la tres grandes ramas hoy día de los sistemas CAD y su geometría subyacente es el modelamiento de los objetos arquitectónicos y productos industriales; La visión artificial y sistemas de obtención de datos por sensores remotos como lo son el reconocimientos de objetos y la fotogrametría; y finalmente el video digital, y sistemas de animaciones digitales son la base de la multimedia y los sistemas de presentación incorporados al web de internet. 
Después de la Segunda Guerra Mundial, el uso de la computación abrió nuevas posibilidades para la teoría de las matemáticas. Los arquitectos hoy día realizan sus trabajos asistidos por programas CAD que han sido los herederos naturales de este desarrollo que comenzó en el renacimiento y que tiene las mismas posibilidades que Monge tuvo en mente cuando desarrolló el sistema de coordenadas cartesianas para dar soporte a la primera gran revolución industrial que el mundo conoció.

\section{Los cambiantes roles del CAD}

El CAD fue introducido en los años '50 para asistir a los diseñadores en la evaluación de los objetivos de sus propuestas. El proceso de diseño de edificios continuó usando métodos manuales, pero a través de la ejecución del diseño, algunas partes fueron desplazadas del proceso manual y llevadas a programas computacionales a fin de realizar diferentes tipos de análisis. El énfasis en las investigaciones en los sistemas CAD derivó desde el mejoramiento de los procesos analíticos, hacia la optimización de las formas generadas mediante el uso de computadores. La representación computacional de los edificios se transformó en el foco central de la investigación. Esta aproximación abrió la compuerta para el desarrollo de los programas CAD, y una gran cantidad de estos programas fueron introducidos en los '70, y llegaron a ser una popular herramienta de diseño en los ' 80 .

La primera generación fue iniciada durante la Segunda Guerra Mundial y estuvo basada en el concepto de «sistemas de análisis», planificando los problemas en una forma racional, secuencial y sistemática, siguiendo pasos específicos o fases. Un analista de sistemas entiende el problema, acopia información, la analiza, genera una solución o soluciones, las implementa, las prueba, $y$, si es necesario, las modifica. La segunda generación de la aproximación a sistemas fue iniciada a fines de los años '60 y estuvo basada en el concepto de «problemas difusos», planificando los problemas en una forma argumentativa. Ambas generaciones de la aproximación a sistemas, aceptan al diseño como una actividad racional ${ }^{9}$. Si se considera a la racionalidad como «pensar antes de actuar», el diseño es sólo parcialmente racional, dado que envuelve tanto sentimientos como pensamiento. De hecho, el diseño es parte del proceso lineal de la vida, la cual es en sí misma un continuo proceso de diseño. El diseño no es «pensar antes de actuar», ni «sentir y pensar antes de actuar», más que eso, es «sentir y pensar al actuar».

\section{¿Qué es el diseño?}

El diseño, en un sentido general, contiene dos conceptos: (a) diseño como un método, y (b) diseño como un tipo de actividad. Ambos conceptos están unidos por una meta común, que es la creación de modelos prácticos. El método del diseño es un procedimiento basado en la formulación de conceptos de estados hipotético de situaciones específicas (generación), haciendo descripciones de estos estados (modelado) y analizando su factibilidad (prueba). Esta terna, generación, modelado y prueba conduce a la formulación de mejores métodos para lograr metas prácticas, es decir mejores medios y más efectivos; en este sentido el método de diseño es el procedimiento para derivar criterios de eficiencia.

Esta especial característica reconoce que la generación de soluciones es una importante propiedad y que la arquitectura es un ejemplo de lo que Simon llamó «solución de problemas en un dominio de tareas semánticamente ricas $»^{10}$

Mientras la representación de los estados de diseño es un problema difícil por sí mismo, la generación de nuevos estados desde uno existente es el problema singular más complejo del diseño arquitectónico, y es este aspecto en el cual los teóricos del diseño están intentando resolver durante esta última década. La mayoría de las herramientas de diseño asistidas computacionalmente (CAD) presumen que los diseñadores trabajan con un problema bien definido. Sin embargo, esta suposición ha sido desafiada por muchos trabajos de investigación en la última década ${ }^{11}$. Los diseñadores comúnmente no tienen una descripción completa del problema antes de comenzar una síntesis del diseño. Durante el diseño conceptual, los diseñadores juegan con ideas para conseguir una mayor comprensión acerca del problema en vez de concentrarse en encontrar simplemente una solución.

\section{Simulaciones espaciales}

La recursividad del proceso generación y prueba establecida como la esencia de las simulaciones integradas en los sistemas CAD permiten resolver el compromiso entre lo que se quiere y lo que se puede. El diseño apunta a producir una descripción potencial de una solución, más que a crearla; los únicos medios para determinar el rendimiento potencial, es a través de una simulación ${ }^{12}$. La simulación del rendimiento puede ser alcanzada de varias formas: a través de dibujos, modelos a escala, cálculos hechos a mano, monogramas, cuadros o algoritmos computacionales. La simulación procede a determinar los valores de las variables de rendimiento que son usados a varios grados de detalles. Ellos van desde simples y relativamente fáciles de producir, apropiadas para rápidas estimaciones del orden de magnitud (bosquejos, modelos a escala, cálculos simplificados), a otras más complejas, relativamente más elaboradas que son altamente detalladas y precisas (dibujos trabajados, modelos a escala detallados, cálculos sofisticados). El proceso de diseño es equivalente a la exploración de lo que es posible hacer bajo un contexto específico y ajustando el criterio de rendimiento de acuerdo a ello, dado que lo que es deseado puede que resulte imposible (ejemplo: Cero costos).

\section{Evaluación multicriterios}

Hay dos aproximaciones principales al considerar la ramificación de criterios cuantitativos de un criterio deliberado. Una es a través de la consideración de un espacio multidimensional definido a través de rangos de valores aceptables para cada criterio cuantitativo, y la otra es a través de la consideración de un índice, que es una función de todas las ramificaciones de criterios cuantitativos ${ }^{13}$. Las versiones más sofisticadas y elaboradas son los modelos de evaluación de multicriterio, los que requieren la especificación de funciones de transformación, de los valores de todas las variables de rendimiento terminales, en valores de lo apropiado o bueno, medidos en

\footnotetext{
Pohl, J., Chapman, A. «An expert design generator». Architectural Science Review 1998; 31

Simon, H. The sciences of the artificial. $2^{a}$ ed. Cambridge, MA, EE. UU. The MIT Press, 1981.

Simon, H. The sciences of the artificial. ${ }^{2}$ ed. Cambridge, MA, EE.U ..: The MIT Press, 1981.

1 Gero, J.S. «A model of designing that includes its situatedness». En: Gu, J., Wei, Z. Proceedings of CAADRIA. 1999.

Scheer, D.R. AlA Building information modeling: What about architecture? EE.UU.: College of Architecture + Planning University of Utah Press, 2006.

13 Papamichael, K.M. «Design process and knowledge: Possibilities and limitations of computer-aided design». Ph.D. Dissertation. Berkeley: University of California, 1991.
} 
una escala común para todas las variables de rendimiento. Además, tales modelos requieren la especificación de la importancia relativa de todas las ramificaciones de criterios de cada juicio deliberado a través de la asignación de factores de peso, y la especificación de una función de agregación donde a través de ella, la variable de rendimiento es computada.

\section{Diseño colaborativo}

El diseño colaborativo asistido por computación (CSCW) se ha estudiado intensamente en reciente años. Los niveles de la infraestructura que habilita técnicamente, como compartir datos, el control de concurrencia y la coordinación de mando, etc., han sido realizados con frecuencia. Pero el aspecto más relevante es la complejidad del diseño, como proceso altamente organizado. Un sistema de CAD colaborativo debe proveer mecanismos para la organización del proceso de diseño. Dentro de la organización, un modelo para el procedimiento de la colaboración necesita de herramientas de apoyo en estas áreas ${ }^{14}$.

La colaboración entre diseñadores puede ser dividida en dos niveles: la colaboración del proyecto y colaboración del diseño. La colaboración del proyecto significa que los diseñadores están trabajando en el mismo proyecto, están compartiendo los mismos datos del plan y están trabajando bajo la misma jerarquía de dirección. Ellos deben trabajar hacia la misma meta y deben terminar su trabajo de acuerdo al mismo calendario. Requiere un mecanismo de organización de proyecto. La colaboración del diseño significa que los diseñadores necesitan seguir ciertos procedimientos durante su colaboración para resolver un problema específico. En este contexto, emerge la necesidad de profundizar en el conocimiento de las tipologías como un posible camino confiable para aproximarse al saber de la arquitectura, desde su propia especificidad y desde su propia razón ontológica.

\section{Geometría y nuevas tecnologías}

El Modelado geométrico trata la representación de formas de cara a visualizarlas, analizarlas, diseñarlas y estudiar su comportamiento.
Todo ello exige adaptarse a los métodos existentes de medición y de toma de muestras, cuando se trata de objetos dados, o adaptarse a los requisitos de los sistemas de manipulación cuando las formas se están creando. El método se deriva directamente de estrategias conocidas de autoorganización bajo aprendizaje competitivo no supervisado, conocida como mapas autoorganizados, representados por grafos y redes neuronales. El grafo mismo se convierte en una red de aprendizaje. Esto es, la red neuronal construye su propia metaheurística a partir de un mecanismo conexionista impulsado por la coherencia del input. El diseño conexionista provee de una flexibilidad casi ilimitada para visualización en una variedad de espacios ${ }^{15}$.

El atractivo de los modelos de autoorganizados (ejemplo: autómatas celulares), radica en que las modelados realizadas muestran patrones espaciales a partir de comportamientos locales (ejemplo: diagramas de Voronoi). El análisis de la dimensión fractálica de la forma superficial y de sus límites resultan ser parámetros que permiten ajustar los patrones globales surgidos de los resultados locales, en este sentido, el análisis de la geometría fractal y la aplicación de autómatas celulares permiten un modelado completo en diferentes escalas de análisis.

\section{Información para la construcción}

La información relacionada con la industria de la construcción es incremetalmente suministrada a los diseñadores y arquitectos directamente en formato digital de tal manera que es posible incorporarla directamente en el proceso de diseño, ya sea como parte de las características y especificaciones del objeto de diseño así como de criterio de evaluación acerca de la efectividad de la solución. De esta manera se pueden reconocer diferentes formas de administrar la información que es suministrada en línea durante el proceso de diseño, la que puede estar ya sea como catálogo, bibliotecas, objetos de bibliotecas, o simplemente como primitivas a instanciar en el proyecto, como tipologías de muros, marcos de puertas y ventanas, cubiertas, escaleras, y los cientos de elementos de infraestructura, sean estos sanitarias, de acondicionamiento físico, eléctricas o de redes de comunicación de datos y seguridad. Estos productos generalmente son suministrados directamente por la industria o incorporados por medio de plug ins dentro de los sistemas CAD ${ }^{16}$.

\section{Arquitectura digital}

La existencia o no de una arquitectura digital, es un tema de debate para críticos e historiadores; los que se han involucrado en el desarrollo de aplicaciones y analizado el efecto de las tecnologías en los modo de producción de la arquitectura, pueden reconocer lo característico de los procesos de camblo, motivados por inversiones tecnológicas. Constituyen elementos estratégicos para la industria y el mercado, ya que posibilita la innovación y nuevas formas de competencia. Ya lejos de aquellos discursos teóricos formalistas, desapegados de la filosofía de la ciencia, que nos relatan los procesos de desmaterialización de la arquitectura, identificando la idea de proyecto como una virtualidad y no como una anticipación, una simulación, un expediente genético que desarrollará un algo material, no podrán reaccionar frente a un nuevo hito del desarrollo de la creatividad humana. Lo cierto es que hoy podemos apreciar exploraciones mediante las cuales se pueden generar espacios definidos por diferentes geometrías, y basados en diferentes transformaciones, es decir: geometrías no euclidianas, sistemas cinéticos y dinámicos, sistemas evolutivos, etc. ${ }^{17}$.

Las formas generadas digitalmente son expresión de una aproximación renovada a la arquitectura, en la que convenciones estéticas o de estilo son ignoradas en favor de una experimentación constante basada en la generación digital y la transformación de formas que respondan a contextos complejos e influencias funcionales. La arquitectura digital es simultáneamente, efecto y expresión de la Revolución de la Tecnología de la Información. Según Manuel Castells, se trata de «una revolución tecnológica, centrada en torno a las tecnologías de la información, que está modificando la base material de la sociedad a un ritmo acelerado» ${ }^{18}$

\footnotetext{
14 Gero, J.S. Op. cit.

15 Klein, M. Capturing geometry rationale for collaborative design. Wetice, 1997.

Scheer, D.R. Op. cit.

Kolarevic, B., Schmitt, G., Hirschberg, U., Kurmann, D., Johnson, B. An experiment in design collaboration. Automation in Construction. 2000.

18 Castells, M. La era de la información economía, sociedad y cultura. Versión castellana de Carmen Martínez Gimeno. Madrid: Alianza Editorial, 1996.
} 


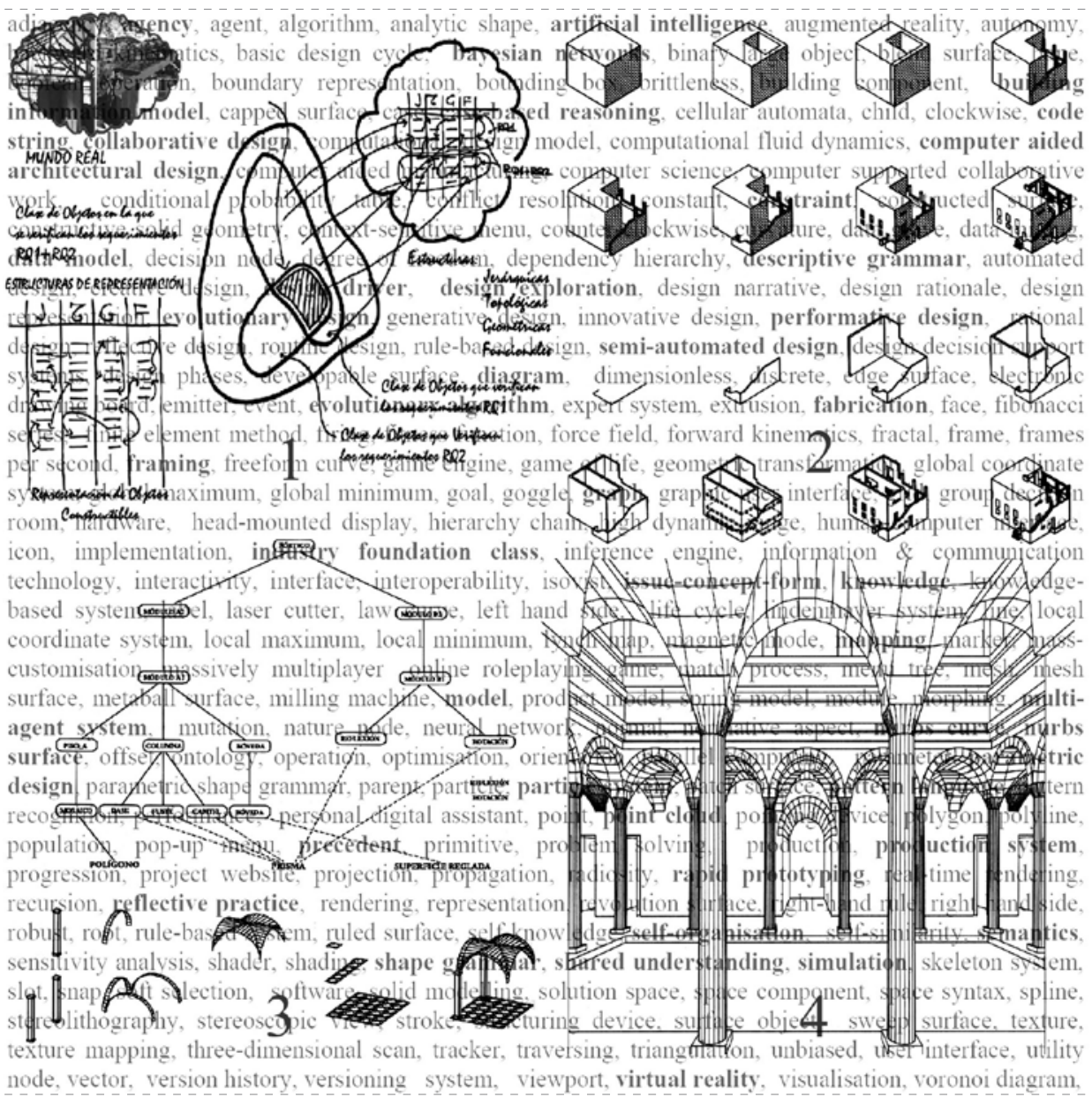

\section{Conclusiones}

Son múltiples las dimensiones educativas que se relacionan con el proceso de modernización. La rapidez y ubicuidad de estos continuos cambios o innovaciones exigen nuevas respuestas de la educación: nuevos contenidos, nuevas instituciones y nuevas formas de aprendizaje. Es necesaria una mayor flexibilidad y adaptabilidad en las funciones que desempeña el sistema educativo para poder contribuir creativamente al desarrollo económico y social en un contexto de cambio continuo y acelerado.

Las nuevas tecnologías requieren nuevos conocimientos en matemáticas, lenguajes simbólicos y geometría, así como nuevas capacidades intelectuales de formalización y abstracción, debido a la creciente importancia del modelado y de la simulación como instrumentos en numerosos campos de trabajo. Así mismo, es importante el análisis lógico relacionado con la elaboración de procedimientos sistematizados, a partir de lenguajes especializados. Las nuevas tecnologías requieren un pensamiento interactivo y divergente, apto para la solución de problemas nuevos y complejos, en lugar de uno lineal y convergente, adecuado para trabajos altamente estructurados y rutinarios.

La profesión de arquitectos es consecuencia de su evolución histórica y en consecuencia,
1 Bosquejo de estructura de datos Frame (Minsky, 1974), que sirve para representar una situación estereotipada de diseño (según el autor 1987).

2. Análisis de dos formas de modelado geométrico

a. Geometría de Construcción de Sólidos (CSG)

b. Modelado de Superficies (poliédricas). Trabajos de estudiantes sobre la obra House on the Lido por A. Loos (1997)

3. Estructura de modelo orientado a objeto de objetos geométricos de diseño (según el autor 20011

4. Modelamiento paramétrico utilizando programación con componentes geométricos (según el autor, scripting GC de Bentley 2007).

Fondo de imagen: Keywords de investigaciones publicadas por organización Cadfuture de Delf University de los últimos 10 años (recopilación del autor). las alteraciones sustanciales producidas en la revolución científica y tecnológica, no han penetrado aún la práctica profesional en profundidad suficiente como para producir variaciones paradigmáticas de la misma. En la Universidad, si no incluimos dicho cuestionamiento en las instancias de docencia y de investigación, estaremos limitándonos a reproducir las opciones tecnológicas ya establecidas. Debemos tomar el liderazgo en el desarrollo de nuevas formas de diseñar, administrar y renovar la infraestructura requerida para soportar las nuevas exigencias de calidad y sustentabilidad demandadas por la sociedad actual. 